\title{
Atuação do psicólogo nos hospitais e nas maternidades do estado de Sergipe
}

\section{The role of the psychologist in hospitals and maternity wards in the state of Sergipe}

Lyvia de Jesus Santos $^{1}$

$M$ aria Jésia Vieira ${ }^{2}$
${ }^{1}$ Universidade Federal de Sergipe. Rua H onor Gregório Santos, 98/ Conjunto LeiteN eto, Grageru. 49027-130 Aracaju SE. Iyviapsico@hotmail.com 2 Programa de Pósgraduação em Ciências da Saúde, U niversidade Federal deSergipe.
Abstract This article seeks to reflect on the professional activity of the psychologist in the hospital context by examining the role of psychologists working in hospitals and maternity wards in the State of Sergipe. It seeks to identify the specific role of these professionals in hospitals and maternity wards, as well as their motivating forces and the difficulties encountered. This work is part of a broader project that sought to study not only the activity per se, but also training aspects of these professionals. The sample was analyzed using a qualitative and quantitative approach for thematic analysis. Results revealed that the characterization of the role of psychologists has a focus on psychotherapeutic work with patients before and after surgery, as well as the caregivers and family members of critically ill patients in the following units: ICU, ICC, oncology, dialysis and surgical wards, offering support, especially at the pre and post-surgery phase.

Key words Psychology, Activities, Hospital psychology, Professional practice, Health work
Resumo 0 presente artigo busca refletir a prática profissional do psicólogo hospitalar, tendo como propósito analisar a atuação daqueles que trabaIham em hospitais e maternidades do Estado de Sergipe. Entender, portanto, as especificidades na atuação deste profissional nos hospitais e maternidades, bem como seus fatores propulsores e suas dificuldades de atuação. Este trabalho é parte de um projeto maior que procurou estudar não só a atuação, mas também os aspectos da formação. A amostra foi analisada na abordagem quali-quantitativa na análise temática. Como resultado, a caracterização da atuação dos psicólogos revelou um enfoque no trabalho psicoterápico junto aos pacientes no préepós-cirúrgico, aos acompanhantes eaos familiares de pacientes críticos internados nas unidades (UTI, CTI, oncologia, hemodiálise e enfermarias cirúrgicas) oferecendo suporte, principalmente em atendimento pré e pós-cirúrgico. Palavras-chave Atuação em Psicologia, Psicologia hospitalar, Prática profissional, Trabalho em saúde 
Introdução

Os primeiros movimentos da atuação de psicólogos em hospitais datam do inicio do século XIX, quando diversos laboratórios de Psicologia instalados em hospitais (psiquiátricos) se faziam presentes. É importante ressaltar que desde a década de 1940 o modelo da política de saúde no Brasil é centrado no hospital ${ }^{1}$.

Os núcleos para uma formação especializada começaram a se difundir nas regiões sul e sudeste do país, eapareceu uma crescentedemanda degraduandosede profissionais com interessenessaárea. $N$ a década de 1990, houve o crescente reconhecimento do psicólogo na instituição de saúde por sua importância na equipe multiprofissional ou interprofissional no atendimento ao pacientehospitalizado. Devido a essa abertura do mercado de trabalho na área de saúde, o setor público tornouse atrativo para os psicólogos, ao oferecer empregos assalariados estáveis. Esses profissionais contratados desenvolvem seus trabalhos, principalmente, nos departamentos de oncologia, cardiologia, CTI eUTI, com pacientes renais, portadores de Síndromedalmuno-DeficiênciaAdquirida(AIDS), suicidas eno atendimento pré-cirúrgico, destacando, ainda, o acompanhamento das parturientes e acolhimento aos recém-nascidos, dentre outros, em maternidades ${ }^{2-6}$.

A pesar da importância da atuação do psicólogo dentro do ambiente hospitalar, a formação deste profissional ai nda está voltada para a clínica centrada no indivíduo, com objetivos psicoterapêuticos, psicodiagnósticos e analíticos. Sua inserção, sem o devido preparo, em ambiente hospitalar, favorece o uso do falso saber, dificultando a comunicação no contexto da instituição, inviabilizando o dinamismo na relação entre equipe de saúde e doente ${ }^{1,7-11}$.

As instituições de saúde, dentro dos seus princípios biomédicos, têm questionamentos sobre o que faz o psicólogo no hospital. N este sentido, o desafio do psicólogo hospitalar é unir o objetivo ao subjetivo, tratar o geral e o particular como forças complementares e não excludentes, a fim de minimizar o sofrimento provocado pela hospitalização bem como diminuir as sequelas emocionais causadas pelo adoecimento e pela hospitalização.

O leque da atuação eintervenção se amplia a cada nova demanda. No ambiente hospitalar 0 profissional esbarra em elementos não controláveis como na clínica, pois além do pacienteterapeuta, há uma equipe inteira, a qual é preciso se integrar. Este paciente hospitalizado faz parte deum contexto institucional, com seus objetivos específicos, da doença e sua cura. Integrar-se a essa equipe, com uma nova especificidade, possibilita uma visão mais global do indivíduo não focada na doença e sim no indivíduo doente ${ }^{2,9,10}$.

Alguns autores apontam o modelo multidisciplinar como o mais adequado à inserção da Psicologia no ambiente hospitalar. Sua articulação cria sistemática para o trabalho, e os profissionais de saúde são levados a reconhecer a necessidade de diferentes contribuições profissionais no cuidado ao paciente de maneira eficiente e eficaz. D esta forma, o foco da doença é deslocado para uma visão mais integrada, embora interdependente e dinamicamente voltados para 0 objetivo comum ${ }^{12,13}$.

As práticas de saúde no modelo biopsicossocial refletem a necessidade de formas mais humanizadas de lidar com o sofrimento e a doença do indivíduo. Desde modo, a Psicologia seinsere na intervenção à saúdeem pequenos passos. Críticas ao modelo biopsicossocial são apontadas, demonstrando que o desafio de inserção interdisciplinar do psicólogo naárea de saúde privilegia a etiologia biopsicossociológica, construindo novas ações de trocas de saberes entre usuários e profissionais $s^{4,6,14}$.

Castro e Bornholdt ${ }^{1}$ sintetizam as funções básicas do psicólogo em atuação hospitalar de acordo com os estudos de Rodriguez-M arín, de 2003: coordenação, ajuda na adaptação, interconsulta, enlace, assistência direta e gestão dere cursos humanos.

Para Sebastiani e M aia ${ }^{6}$, o psicólogo atua como minimizador da angústia e da ansiedade do paciente, "favorecendo a expressão dos sentimentos e auxiliando na compreensão da situação vivenciada", proporcionando verbalização das fantasias, bem como reforçando a confiança entre paciente e equipe de saúde. Faz-se necessário, principalmente, a atuação na reorganização do esquema da consciência do pacienteno mundo e sua imagem corporal, necessários para a reestruturação do autoconceito.

As Diretrizes Curriculares N acionais ${ }^{15}$ (DCN) para o curso de Psicologia, do ano de 2004, contemplam uma formação ampla do psicólogo, trazendo habilidades e competências a serem aplicadas à prevenção e promoção da saúde, tendo como garantia de identidade do curso o núcleo comum que assegura a base homogênea para a formação e a capacitação. Visam ainda garantir ao profissional o domínio de conhecimentos psicológicos e a capacidade de utilizá-los em diferentes contextos ${ }^{15}$. 
Em Sergipe, os primeiros estudos na área de psicologia foram realizados por médicos, tendo como registro mais antigo o trabalho datado de 1858, sobre o tratamento de doenças mentais. No início do século XIX, teses defendidas por sergipanos na Faculdade de Medicina da Bahia, e mais tarde um estudo sobre aerofagia histérica, em 1902, e outro sobre fobias, em 1904, são os registros que demonstram o interesse pelo campo psicológico ${ }^{16}$.

0 primeiro curso de graduação em Psicologia no Estado deSergipe data de 1990 em universidade pública, seguido deuma instituição particular em 1996, eo terceiro em 199917. Em 2008 foi criado mais um curso de Psicologia no Estado.

A atuação do profissional na área hospitalar começou aser definidaa partir da inserção em dois hospitais psiquiátricos da rede pública do Estado, na década de 1980, estendendo-se para particulares, também psiquiátricos. No tocante à rede de hospitais gerais, a inserção desse profissional só se deu no ano de 1991, época em que o curso de graduação em Psicologia da Universidade Federal de Sergipe completava seu primeiro ano ${ }^{17}$.

Atualmente, com a visão interdisciplinar do fenômeno saúde-doença, o pacienteévisto como um todo na situação em que ali se encontra. E essa visão interdisciplinar se faz importante nos hospitais, pois os pacientes apresentam uma dimensão, por vezes, física, biológi ca epsíquica em desestabilização, caracterizados por um desequilíbrio biopsicossial. Envolve assim, diversas manifestações somáticas e psíquicas desde a admissão até a alta hospitalar, 12,13,18.

O suporte psicológico deve ser realizado a todos, paciente, familiar e membros da equipe de saúde que demandarem este tipo de apoio e para auxiliar no tratamento e na compreensão da internação, da doença ou do adoecimento, bem como de óbito em vias de elaboração da perda.

Entretanto, as instituições de saúde, dentro dos seus princípios biomédicos, têm questionamentos sobre o que faz o psicólogo no hospital. Pode-sedizer queseu desafio éunir o objetivo ao subjetivo, tratar o geral e o particular como forças complementares e não excludentes, a fim de minimizar o sofrimento provocado pela hospitalização bem como diminuir as sequelas emocionais causadas em decorrência do adoecimento e da hospitalização9.

O leque da atuação e intervenção se amplia a cada nova demanda. $\mathrm{Na}$ Psicologia Hospitalar lida-secom elementos não controláveis, pois além do paciente-terapeuta, trabal ha-secom uma equipeinteira, eé preciso se integrar a essa equipe. 0 paciente hospitalizado faz parte de um contexto institucional com seus objetivos específicos. Integrar-sea essa equipe, com uma nova especificidade, possibilita uma visão mais global não focada na doença e sim no indivíduo doente $7,9,12,13,18$.

As práticas de saúde no modelo biopsicossocial refletem a necessidade de formas mais humanizadas de lidar com o sofrimento e a doença do indivíduo. Com esse novo modelo, a Psicologia se insere na intervenção à saúde em pequenos passos. Críticas ao modelo biopsicossocial são apontadas, demonstrando que o desafio de inserção interdisciplinar do psicólogo naárea de saúde privilegia a etiologia biopsicossociológica, construindo vertentes políticas de novas ações de trocas de saberes entre usuários e profissionais $s^{4,6,14}$.

Entender, portanto, as especificidades da atuação deste profissional nos hospitais e maternidades, bem como seus fatores propulsores e dificuldades de atuação foi o foco deste trabalho que é parte de um projeto mai or que procurou estudar não só a atuação, mas também os aspectos da formação destes.

\section{Métodos}

Trata-se de um estudo descritivo de caráter exploratório de abordagem quanti-qualitativa, em que foram investigados 29 profissionais de psicologia que atuam em hospitais e maternidades do Estado de Sergipe. Foram excluídos da pesquisa psicólogos que atuam em hospitais psiquiátricos ou em setores administrativos da instituição, por terem focos diferentes de atuação. Os dados foram coletados por meio de um questionário misto. Foram investigadas questões referentes à sua atuação e formação. Para fins deste trabal ho estão sendo utilizados apenas os dados referentes à atuação.

Foram inicialmente identificadas as instituições que tinham em seu quadro funcional psicólogos atuando na assistência hospitalar, resultando em três $M$ aternidades, sendo uma fundação, uma pública e uma particular; um Hospital-maternidade, particular; e dez hospitais, sendo uma fundação, oito públicos (um federal, seis estaduais e um municipal) e um particular.

Todos os indivíduos que faziam parte destas instituições e que aceitaram participar da pesquisa foram incluídos e receberam orientações verbalmente e por escrito a respeito dos objetivos e de seus direitos, assinando, em seguida, o termo de consentimento livree esclarecido. Todo o estudo foi conduzido de acordo com os princí- 
pios éticos regidos pelas Diretrizes e Normas Regulamentadoras de Pesquisa Envolvendo Seres Humanos, segundo a resolução 196/1996 do Conselho Nacional de Saúde, Brasil. 0 projeto foi aprovado pelo Comitê de Ética em Pesquisa Envolvendo Seres Humanos da Universidade Federal deSergipe (CEP/UFS).

Os dados coletados forma analisados em escala ponderal, nas questões fechadas. Já para as respostas das perguntas abertas, procedeu-se a análise qual itativa na abordagem temática baseado em Bardin ${ }^{19}$ e Minayo ${ }^{20}$.

A escala ponderal utilizada por Vieira ${ }^{21}$ subsidiada em Good e Hatt destina-se a transformar atributos qualitativos em variáveis quantitativas. Consiste em atribuir pesos para valoração de atributos pelos sujeitos, a fim de encontrar o escore (E) que demonstra a ordem de valoração atribuída à variável de maior valorização, e assim sucessivamente. N este trabalho, a partir da ordenação atribuída pelos respondentes aos três tipos de atendimentos mais frequentes na prática hospitalar, foi atribuído o peso 3 para o mais frequente, peso 2 para o $2^{\circ}$ em ordem defrequência e peso 1 para o 30 em sequência. 0 número de respostas $(\mathrm{N}$ ) foi multiplicado pelo peso da ordem de frequência correspondente, obtendo-se o escore total apurado para cada tipo de atendimento referido.

\section{Resultados ediscussão}

Dos profissionais pesquisados, $72,4 \%$ atuam na rede pública, 20,6\% na rede particular e 6,8\% na rede pública e particular. A atuação em instituição pública tem-se dado a partir de contratos e concursos, estes a partir do ano $2002^{17}$. Este fato pode explicar o dado de $55 \%$ deles terem atécinco anos de experiência, demonstrando ser esta uma área de atuação recente.

$\mathrm{N}$ as instituições pesquisadas a atuação mais referida, por ordem de frequência, foi com paciente em período pré cirúrgico, como demonstra a Tabela 1. Estes pacientes têm sido, principalmente, portadores de patologias cardíacas e renais, eaqueles submetidos às cirurgias bariátricas e mastectomias, sendo a intervenção mais citada o suporte nos momentos pré-operatório. 0 suporte pós-operatório foi o terceiro mais citado.

Este resultado tem relação com o início da atuação da Psicologia em hospitais, no Brasil, nos anos 1950, quando Matilde Neder iniciou 0 acompanhamento na reabilitação pós-cirúrgica de pacientes traumáticos, com resultados que fortaleceram a demanda para atuação nesta área².

De acordo com Sebastiani e M aia ${ }^{6}$, o paciente cirúrgico tem intenso desconforto emocional caracterizado como impotência, isolamento, medo da morte, da dor, da mutilação e das mudanças de sua imagem corporal. Para esses autores, nenhum pacienteestá preparado efetivamente para subme ter-seà cirurgia, e acreditam, assim, que a atuação psicológica no período préoperatório influencia diretamente no trans e no pós-operatório.

M esmo considerando o fato histórico da atuação dos psicólogos nos hospitais, no nosso meio esta atuação ainda é recente, o que se relaciona, diretamente, às dificuldades sentidas pelos profissionais e à carência de maiores investimentos das instituições formadoras.

0 atendimento aos acompanhantes foi o terceiro escore apurado, na ordem de frequência, principalmente àqueles que têm familiar portador de doença crônica, internados em unidades deterapiaintensiva(UTI), quenão podem acom-

Tabela 1. Tipos de atendimentos realizados pelos psicólogos na prática hospitalar, por ordem de frequência. Aracaju (SE), 2009.

\begin{tabular}{|c|c|c|c|c|c|c|c|}
\hline \multirow{3}{*}{ Tipos de atendimentos } & \multicolumn{6}{|c|}{ Ordem de citação das atividades mais frequentes } & \multirow{3}{*}{ Total } \\
\hline & \multicolumn{2}{|c|}{$01(x 3)$} & \multicolumn{2}{|c|}{$02(x 2)$} & \multicolumn{2}{|c|}{$03(x 1)$} & \\
\hline & $\mathrm{N}$ & $\mathrm{E}$ & $\mathrm{N}$ & $\mathrm{E}$ & $\mathrm{N}$ & $\mathrm{E}$ & \\
\hline Urgência & 03 & 09 & 01 & 02 & 02 & 02 & 13 \\
\hline Pré-cirúrgico & 09 & 27 & 04 & 08 & 02 & 02 & 37 \\
\hline Pós-cirúrgico & 02 & 06 & 11 & 22 & 03 & 03 & 31 \\
\hline Ao paciente por negação de cuidado & 03 & 09 & - & - & 02 & 02 & 11 \\
\hline Ao acompanhante & 03 & 09 & 07 & 14 & 11 & 11 & 34 \\
\hline À equipe de saúde & - & - & 02 & 04 & 02 & 02 & 06 \\
\hline Ao familiar por falecimento do paciente & 02 & 06 & 02 & 04 & 01 & 01 & 11 \\
\hline Outros & 07 & 21 & 01 & 02 & 02 & 02 & 25 \\
\hline
\end{tabular}


panhá-los a maior parte do tempo nos procedimentos e nos períodos de hospitalização.

0 atendimento de urgência corresponde, na maioria das vezes, a um pedido emergencial deinterconsulta, embora na amostra do estudo não haja profissionais atuando diretamenteem unidadesdeurgênciaeemergência, sendo estes requisitados de outras unidades. Em termos exatos, a intervenção da Psicologia no pronto socorro ocorre após a estabilização das funções vitais do paciente para uma posterior assistência psicológica; auxilia no tratamento dos aspectos psicossociais e na implicação da reabilitação; oferece acolhimento para o paciente, acompanhante/familiar eà equipe.

No estudo de Vieira ${ }^{17}$ foi identificado um único profissional que atuava na urgência do maior hospital público do estado de Sergipe. Dentre os tipos de atendimentos enquadrados como outros, relata-se 0 acolhimento, 0 atendimento ao paciente infantil e ao familiar por outros motivos.

Foi destacado ainda, neste estudo, 0 atendimento ao paciente que se nega a receber algum tipo de cuidado e o acompanhamento específico ao familiar por falecimento de paciente na interconsulta e/ou na abordagem para doação de órgãos e tecidos.

0 atendimento à equipe de saúde também foi citado por quatro respondentes, dois deles como sendo o segundo mais frequente e dois como o terceiro mais frequente.

Agrupados como outros, com escores menores, foram citados os atendimentos ao paciente pediátrico, às gestantes e às puérperas, realizados por interconsulta ou por atuação de equipe multidisciplinar e ainda as visitas domiciliares, quando necessárias.

Em relação às abordagens terapêuticas utilizadas, $18 \%$ dos profissionais declaram utilizar a Psicanálise, $14 \%$ a Fenomenologia e $9 \%$ o Existencialismo. Estas duas últimas teorias vêm se destacando na vertente da Psicologia H ospitalar brasileira, segundo estudos deAngerami-Camon². A Psicossomática, abordada por $11 \%$ dos respondentes, éuma teoria descendenteda M edicina Psicossomática, muito utilizada em hospital22.

Entre outras abordagens referidas por $14 \%$ da amostra, estão incluídas: a Psicodinâmica de base analítica, a Logoterapia (Existencial ista eH umanista), e o Cognitivismo/Comportamental. Ressalta-se que dos 29 pesquisados, 20,6\% responderam que utilizam duas abordagens teóricas e 13,7\% utilizam até três tipos de abordagens.

No estudo de Vieira ${ }^{17}$, a Psicanálise também teve uma frequência semelhante (18\%), seguido do Psicodrama com $17 \%$ e Gestalt com $14 \%$. Bardagi et al..$^{23}$ referiram que a Psicanálise aparece em $42 \%$ e a Psicologia social/institucional em $15 \%$, sendo estas as linhas teóricas mais citadas entre as dez abordagens referidas como base para a prática profissional.

Em relação aos modos de intervenção foram citadas: a psicoterapia breve, focal e de apoio; interconsultas; orientação psicológica; apoio matricial; acolhimento; avaliação de doador e receptor para transplante.

0 apoio matricial é uma forma de organizar e ampliar a oferta de ações em saúde, aumentando a capacidade resolutiva de problemas de saúde pela equipe local, compartilhando responsabilidades e vínculo terapêutico. A composição desses novos arranjos se deve à criação de possibilidades para operar com a ampliação do trabalho clínico, considerando quenenhum especialista, de modo isolado, poderá assegurar uma abordagem integral ${ }^{24}$.

Alguns aspectos foram destacados pelos respondentes quanto à sua prática no trabal ho hospitalar (Quadro 1), alguns deles como facilitadores e outros restritivos à atuação.

$\mathrm{Na}$ relação com o paciente e o familiar, responsáveis pela manuten ção do vínculo assistencial, foram identificados os núcleos de sentido: 'receptividade', 'cooperação' e 'relação empática', esta última referida à questão de afetividade e percepção do paciente, familiar e/ou equipe para com o psicólogo. Esta situação permite ao profissional observar reações emocionais do outro, favorecendo uma maior interação na psicodinâmica do processo terapêutico ${ }^{2,8,13}$.

$N$ a relação do profissional com a instituição, foram identificados os núcleos de sentido: 'disponibilidade deterapêutica'; 'partici pação em diversas atividades' ea 'aplicação do conhecimento acadêmico'.

Neste aspecto, foi referido ser 0 ambiente do hospital crítico para a abordagem terapêutica dos psicólogos, uma vez que funciona 24 horas por dia, tem uma grande demanda de pacientes e, algumas vezes, em conjunto com outros procedimentos de rotina hospitalar, sem garantia de espaço esilêncio.

Penna ${ }^{25}$ e Romano ${ }^{8}$ referem, entretanto, que a partir destas condições esses profissionais adquirem como característica específica 0 aprendizado para a utilização desses espaços intra-hospitalares na tentativa de realizar 0 atendimento adequado aos pacientes e familiares. É preciso que o psicólogo seja flexível e criativo, para contornar as dificuldades ${ }^{25}$. 0 psicólogo está onde 
Quadro 1. Aspectos destacados pelos respondentes quanto à prática do psicólogo no trabalho hospitalar. Aracaju (SE), 2009.

\begin{tabular}{|l|l|}
\hline \multicolumn{1}{|c|}{ Subcategorias } & \multicolumn{1}{c|}{ Núcleo de sentido } \\
\hline Relação com pacientes e familiares & $\begin{array}{l}\text {. Receptividade } \\
\text { - Cooperação } \\
\text {. Relação empática }\end{array}$ \\
\hline Relação do profissional com a instituição & $\begin{array}{l}\text {. Disponibilidade terapêutica } \\
\text {. Participação em diversas atividades } \\
\text {. Aplicação do conhecimento acadêmico }\end{array}$ \\
\hline Organização no processo de trabalho & $\begin{array}{l}\text {. Flexibilidade dehorário } \\
\text { - Tempo de internação } \\
\text {. Liberdade para trabalhar a demanda dos pacientes } \\
\text { - Projetos isolados }\end{array}$ \\
\hline Humanização & $\begin{array}{l}\text { - Interação da equipe multiprofissional } \\
\text { - Relação psicólogo-paciente }\end{array}$ \\
\hline
\end{tabular}

os acontecimentos estão e deve ir ao encontro dos pacientes ${ }^{8}$.

A participação do psicólogo em diversas atividades no hospital, particularmente em grupoterapia, acolhimento e humanização é cada vez mais valorizada pela gestão hospitalar. Esteé um compromisso real que remete à consolidação da psicologia como especialidade nas atividades diárias do hospital, propondo novas maneiras de trabalhar a produção do cuidado da forma mais integral possível. A capacidade de cada profissional exercer a função de "gestor do cuidado" ao usuário relaciona-se com a criação do vínculo e responsabilização do trabalho em equipe. As ações, para tanto, devem garantir a qualidade resolutiva da assistência, tanto como eficácia técnica-científica quanto com adesão e intercomunicação na relação direta entre diversos profissionais e destes com os usuários; além de ter o compromisso de se fazer a melhor escuta possível das necessidades de saúde trazidas por aquelas pessoas que buscam o serviço ${ }^{26-28}$.

Dentre os respondentes que citaram a organização do processo de trabalho como subcate goria, observa-se o lado positivo da instituição hospitalar nosrespectivos núcleos de sentido: 'flexibilidade de horário'; 'tempo de internação' e'liberdade para trabalhar a demanda do paciente'.

$\mathrm{N}$ as estratégias de processo do trabalho hospitalar, o regime de turno de plantão, a permanência do paciente em internação e as necessidades do paciente e familiar, diante da dor e hospitalização, potencializam a ação de fatores que por si só comprometem sua integridade física e psíquica.
N a subcategoria humanização, os núcleos de sentidos encontrados correspondem a: 'interação das equipes multiprofissionais' e'relação psicólogo-paciente'.

A humanização é preconizada pelo M inistério da Saúde como o programa de Política Nacional de Humanização (PNH) - Humaniza SUS, que abrange prevenção, cuidado, proteção, tratamento, recuperação, promoção e produção de saúde $^{29}$. A postura e a prática do acolhimento favorecem a construção de relação de confiança e compromisso dos usuários e equipes de serviços, contribuindo para a promoção da cultura de solidariedade e para a legitimação do Sistema Ú nico de Saúde(SUS).

D estaque-se, como experiência prática o trabalho de humanização que ocorre em um hospital particular de Aracaju, desde o ano de 2003, com referencial teórico da Psicanálise. 0 professor psicólogo e seus estagiários desenvolvem atividades com artes (pintura, música etc.) na assistência à saúde. Este trabalho é realizado com pacientes, acompanhanteseprofissionais desaúde após entrevista prévia para mel hor caracterizar a realidade a ser trabalhada ${ }^{30}$.

A questão da humanização na atenção à saúde se estabeleceem diferentes nuances, transcendendo a noção desaúde, ea integração da equipe de saúde e é imprescindível para que o atendimento e o cuidado alcancem a amplitude do ser humano. Portanto, o trabalho em equipe mostra-se fundamental para a qualidade dos serviços hospitalares, ${ }^{6,31}$. Para Tonetto e Gomes ${ }^{13}$, a pertinência do trabalho do psicólogo no hospi- 
tal depende de deslocamento do foco doença para uma visão integrada do processo saúde-doença, no crescente modelo biopsicossocial. A humanização também parte da necessidade da equipe ${ }^{14}$.

0 Q uadro 2 indica as subcategorias identificadas entre as dificuldades na atuação do psicólogo hospitalar com seus respectivos núcleos de sentido.

Segundo os respondentes, embora tenha havido um crescimento do número de profissionais, principalmentenos hospitaispúblicos, nosúltimos anos, através de contratos e concursos, estenúmero ainda não é suficiente para a necessidade.

Destacam como dificuldades na prática com relação aos pacientes, o 'estado biopsicossocial e indisposição do paciente, interferindo no atendimento,' 'criança refratária ao atendimento' ea'falta de entendimento da prática do psicólogo'.

0 ambiente hospitalar suscita diferentes situações e emoções advindas da patologia e traz implicações que criam dificuldades e limitações ao trabalho desses profissionais.

A criança, muitas vezes refratária ao atendimento, precisa ser inserida demaneira mais agradável possível e o brincar é uma das estratégias de enfrentamento da hospitalização, pois o uso do brinquedo de forma terapêutica tem-se mostrado alternativa eficaz nestes casos, conforme referem, entre outros, M otta e Enumo ${ }^{32}$ e ainda M oraes e Enumo ${ }^{33}$.

Aliado, ainda a estes fatores, os pacientes nem sempre entendem o trabalho do psicólogo, podendo este desconhecimento estar relacionado a aspectos culturais, tais como a visão da psicologia como profissão elitista.

No que diz respeito às dificuldades com relação à equipe de saúde, outros fatores queinterferem na prática do psicólogo na instituição hospitalar, conforme referido pelos sujeitos, são 'interven ções no momento do atendimento'; 'predomínio do modelo biomédico'; 'falta de reconhecimento do trabalho do psicólogo'; e'ausência de interdisciplinaridade'.

Com relação à instituição, embora tenha sido referido por alguns respondentes a valorização das ações do profissional por parte dos gestores, foram destacados por outros o pouco incentivo para o profissional atuar em equipe, a falta de receptividade e compreensão do papel do psicólogo em ambiente hospitalar, como demonstra o relato:

ausência de uma política de reconhecimento e valorização do profissional psicólogo por parte da instituição $(\psi 4)$.

A ausência de uma rotina de solicitação do trabalho psicológico aliado à falta de estrutura, a des-

Quadro 2. Dificuldades na atuação do psicólogo no trabalho hospitalar. Aracaju (SE), 2009.

\begin{tabular}{|c|c|c|}
\hline Subcategorias & & Núcleo de sentido \\
\hline Quadro funcional & \multicolumn{2}{|c|}{. Número reduzido de psicólogos } \\
\hline \multirow{4}{*}{ Dificuldade na prática } & $\begin{array}{l}\text { Com relação } \\
\text { aos pacientes }\end{array}$ & $\begin{array}{l}\text {. Estado biopsi cossocial e indisposição interferem no } \\
\text { atendimento } \\
\text {. Criança refratária ao aten dimento } \\
\text {.Falta de entendimento da prática do psicólogo }\end{array}$ \\
\hline & $\begin{array}{l}\text { Com relação } \\
\text { à equipe }\end{array}$ & $\begin{array}{l}\text { Intervenções no momento do atendimento } \\
\text {. Predomínio do modelo biomédico } \\
\text {. Ausência de interdisciplinaridade } \\
\text {. Falta dereconhecimento do trabalho psicológico }\end{array}$ \\
\hline & $\begin{array}{l}\text { Com relação } \\
\text { à instituição }\end{array}$ & $\begin{array}{l}\text { Falta de uma rotina de solicitações e demandas de } \\
\text { atendimento } \\
\text {. Entraves estruturais } \\
\text {. Pouco incentivo, receptividade e compreensão ao papel } \\
\text { do psicólogo em ambiente hospitalar }\end{array}$ \\
\hline & $\begin{array}{l}\text { Com relação } \\
\text { ao psicólogo }\end{array}$ & $\begin{array}{l}\text { - Adequação ao ambiente de atendimento } \\
\text {. Capacidade detranspor obstáculos relacionadosà } \\
\text { dinâmica hospitalar/equipe } \\
\text {. Descontinuidade do tratamento }\end{array}$ \\
\hline
\end{tabular}


valorização do profissional ea outros entraves que permeiam a organização também foram abordados como dificuldades com relação à instituição.

O hospital éuma instituição deal ta complexidadee a falta de clareza diante de profissões emergentes dificulta o trabalho em equipe ${ }^{13}$. M ogano, no Seminário sobre Profissões de Saúde, enfatizou a valorização e reconhecimento do psicólogo por outros profissionais como de fundamental importância para o trabalho interdisciplinar ${ }^{34}$.

O Fórum Nacional de Psicologia e Políticas Públicas ${ }^{35}$, no tocanteà inser ção do psicólogo na unidade hospitalar, propôs a obrigatoriedade do profissional dePsicologia nas equipes permanentes deUTI, serviços de aten ção a pacientes renais crônicos, cardíacos eoutros ena composição das Comissões Intra-H ospitalares de D oação de Ó rgãos e Tecidos para Transplantes (CIHDOTT). Cabe aos Sistemas de Conselhos de Psicologia elaborar, em parceria com entidades cientificas da área, diretrizes e protocolos de atuação do psicólogo nestes ambientes ${ }^{35}$.

No trabal ho hospitalar, as diversas categorias profissionais são contribuintes e complementares; não é algo isolado e fragmentado. Mas, um respondente relatou ter melhor desempenho do serviço prestado quando o faz de forma isolada:

(...) 0 isolamento entre os profissionais das varias disciplinas resulta em uma maior liberdade para desenvolvimento de quaisquer projetos (fora da perspectiva interdisciplinar) ( $\psi 05)$.

Os Conselhos de Psicologia fazem gestão a fim de garantir a inserção do psicólogo na equipeinterdisciplinar, ampliar edivulgar o trabalho do psicólogo no atendimento domiciliar a pacientes em cuidados paliativos em alta da rede hospitalar e a pacientes em tratamento domiciliar ${ }^{35}$.

Com relação às dificuldades no que diz respeito à sua própria atuação, observou-sequepara alguns respondentes no âmbito hospitalar não existem facilidade para atuação, o que impõe a esses profissionais a capacidade de transpor obstáculos institucionais conforme relato:

Vocêtem que se adequar ao ambiente para um melhor atendimento junto ao paciente e familiar ( $\psi 10)$.

Foi investigada, ainda, neste trabalho, a percepção dos respondentes sobre as competências necessárias ao profissional para a atuação hospitalar (Quadro 3).

Tonetto e Gomes ${ }^{13,36}$ estudaram as habilidades e as competências adquiridas pelos psicólogos hospitalares. Segundo eles, espera-se que 0 psicólogo no hospital seja capaz de desenvolver ações de assistência, ensino e pesquisa, e o desenvolvimento destas habilidades ecompetências, na sua formação, visa proporcionar maior interação entre teoria e prática, utilizando da competência como padrão dequalidadequese refleteno procedimento real e consensual do desempenho.

As competên cias e habilidades necessárias ao exercício do psicólogo estabelecidos pelas Diretrizes Curriculares Nacionais ${ }^{15}$ para a Graduação de Psicologia (Resolução CNE/CES no 8, de 07/05/2004), referem-se: a) ao domínio básico de conhecimentos psicológicos e uma formação ampla que contempla a multiplicidade de suas concepções teóricas e metodológicas; b) à diver-

Quadro 3. Percepção dos respondentes sobre as competências necessárias para atuação hospitalar. Aracaju (SE), 2009.

\begin{tabular}{|c|c|c|}
\hline Subcategoria & & Núcleos de sentido \\
\hline \multirow{4}{*}{ Competências na prática } & $\begin{array}{l}\text { Com relação ao paciente e } \\
\text { familiar }\end{array}$ & $\begin{array}{l}\text {. Comunicação } \\
\text {. Dinamismo } \\
\text {. Segurança }\end{array}$ \\
\hline & Com relação à equipe & $\begin{array}{l}\text { Observância de princípios éticos } \\
\text {. Habilidade para trabalhar em equipe }\end{array}$ \\
\hline & Com relação à instituição & $\begin{array}{l}\text { Saber lidar com o ambiente hospitalar } \\
\text {. Flexibilidade }\end{array}$ \\
\hline & Com relação ao psicólogo & $\begin{array}{l}\text { Interesse pelo trabalho } \\
\text {. Determinação } \\
\text {. Desenvolvimento pessoal } \\
\text {. Iniciativa } \\
\text { Tolerância à frustracão }\end{array}$ \\
\hline
\end{tabular}


sidade de suas práticas e contextos de atuação; e c) à capaci dade de utilizá-los em diferentes contextos adequados à demanda e à população-alvo em termos individuais e coletivos.

Conceber um currículo com competências e habilidades determina o encontro do estudante com o conhecimento, utilizando-sedeprocedimentosclaramentedefinidos ${ }^{13}$. A competência éo fator mais importante para o papel desempenhado pelo profissional em seu ambiente de trabalho ${ }^{37-39}$.

Para a formação dos psicólogos que atuam especificamente nos hospitais, Besteiro e Barre to ${ }^{40}$ afirmam queéindispensável treinamento em três áreas: clínica ( realizar avaliações e intervenções psicológicas) , pesquisa (saber conduzir pesquisas e comunicar informações psicológicas a outros profissionais) e a programação (desenvolver habilidades para organizar e administrar programas em saúde).

Quanto aos pacientes e familiares, as competências apontadas pelos respondentes como necessárias para atuação junto a estes foram: 'comunicação', 'dinamismo' e'segurança'. Alguns respondentes apontam a observância dos princípios éticos e a habilidade para trabal har em equipe. Convém ressaltar que esse último também é apontado como um dos fatores de dificuldades de atuação.

As Diretrizes Curriculares (2004) prevêem a atuação do psicólogo inter e multiprofissionalmente e a exploração das interfaces com outras áreas, e levam em conta a expansão da atuação profissional para novos contextos, além de acentuar a dimensão social e a ética profissional.

N este campo de formação (hospitalar), a psicologia tem a oportunidade de desenvolver as competências para o exercício do trabalho em equipe, com troca de experiências e saberes contribuindo para compreensão mais integral do indivíduo.

Ainda como competências necessárias para a prática, os respondentes refletem sobreas relacionadas asi mesmo no que diz respeito ao 'interesse pelo trabalho', à'determinação, ao desenvolvimento pessoal, à iniciativa, à tolerância à frustração' e à necessidade de 'submeter-se à terapia'.

A cada ano, o número de adeptos à Psicologia Hospitalar vem crescendo, e no Estado de Sergipe dois cursos de pós-graduação já foram instalados. A presença do psicólogo na equipe do hospital é de fundamental importância, pois as elaborações subjetivas podem ser detectadas e trabalhadas, melhorando o processo de hospitalização para a reintegração à vida. Cabe ao psicólogo conquistar pelo seu conhecimento e por sua dedicação o seu espaço nas equipes de saúde.
N este sentido, é importante a adequada formação para atuar na saú de, seja nos hospitais ou em qualquer outro ambiente que trabal he os aspectos da promoção de saúde na população, acreditando que as mudanças devem se iniciar nos espaços de formação, no intercâmbio tanto entre os membros da equipe de profissionais, como na relação profissional/paciente-usuário do serviço.

Sabendo que a reformulação das Políticas Públicas em Saúde é fruto de conquistas impostas pelas demandas sociais e que urge um posicionamento adequado do psicólogo frente às necessidades do trabalho em saúde (hospitalar), espera-se que a proposta do presente trabalho contribua com reflexões equestionamentos, bem como, seja útil ao desenvolvimento de ênfases na proposta pedagógica dos cursos de graduação e pós-graduação em Psicologia.

\section{Consideraçõesfinais}

O presente estudo descreveu as demandas e os aspectos da atuação do psi cólogo, nas várias atividades que compreendem a sua prática hospitalar, atendendo, portanto, aos seus objetivos.

A inserção do psicólogo na rede de saúde, em particular nos hospitais e maternidades, tem importância para o paciente diante da enfermidade, minimizando o sofrimento provocado pela perda da saúde, pelo isolamento familiar e sua relação biopsicossocial. As possibilidades de intervenções envolvidasformam um entrelaçamento com outras profissões da área de saúde trazendo como compreensão o indivíduo como um todo: universal, integral eúnico, como preconiza - Sistema Ú nico de Saúde do Brasil.

O tempo de inserção do psicólogo, observado nos hospital e maternidades estudadas é relativamente pequeno e há um contingente reduzido de profissionais atuando na área. 0 contingente maior é de profissionaisjovens e do gênero feminino, tendo a psicanálise como abordagem psicoterapêutica, em sua maioria.

A caracterização da atuação dos psicólogos revelou um enfoque no trabalho psicoterápico junto aos pacientes no prée pós-cirúrgico e, principalmente, aos acompanhantes efamiliares, de pacientes críticos internados nas unidades (UTI, CTI, oncologia, hemodiálise e enfermarias cirúrgicas).

Foram identificadas dificuldades em articular a Psicologia no corpo clínico no contexto hospitalar, diante das limitações estruturais ou mesmo da inabilidade para se trabal har no hospital. 
É possível perceber, a partir das informações dos participantes, a fragilidade no trabalho interdisciplinar eno reconhecimento da função do psicólogo no âmbito hospitalar, gerando, assim na percepção destes, dificuldades de consolidação do trabalho.

Os dados obtidos reforçam a necessidade de trabalho em equipeinterdisciplinar em saúde, no sentido de instaurar um modelo efetivamente biopsicossocial do processo de saúde, em que diversas abordagens possam contribuir para a diminuição de riscos e o aumento da qualidade do atendimento e de vida dos pacientes e familiares.

A intervenção realizada pelo psicólogo se dá com confrontos entreteoria e vivência profissional na rede de saúde e resulta na capacidade de buscar alternativas e estratégias de superação frente a impasses e dificuldades. Tais dificuldades estão relacionadas principalmente: a) ao seu preparo que expressam nas necessidades de meIhorar sua qualificação profissional, competên- cias e habilidades; b) à deficiência na interlocução e ao trabalho fragmentado quando diz respeito à interação com os demais membros da equipe interdisciplinar engajada na prestação do cuidado ao paciente; c) ao não reconhecimento dafunção desempenhada pelo psicólogo na equipecomo principal fator impeditivo para a execução do seu trabalho.

Depreender-se daí que face à expansão do trabal ho na área hospitalar, econsiderando as dificuldades referidas, tanto no que diz respeito ao seu preparo quanto ao reconhecimento de suas funções, urge a necessidade de que as instituições formadoras invistam em um ensino que prepare os profissionais para o desempenho da assistência hospitalar. De igual forma, énecessário que as instituições de saúde e seus órgãos gestores, face à necessidade de atuação destes profissionais, invistam no reconhecimento do seu papel perante a equipe, propiciem a sistematização desta assistência e a real integração da equipe multiprofissional.

\section{Colaboradores}

LJ Santos e MJ Vieira participaram igualmente de todas as etapas de elaboração do artigo.

\section{Agradecimentos}

Ao M estre Diego Noronha de Góis, pelo incentivo e contribuição. 
1. Castro EK, Bornholdt E. Psicologia da saúde x Psicologia Hospitalar: definições e possibilidades de inserção profissional. Psicol Cien Prof 2004; 24(3):48-57.

2. Angerami-Camon VA. Psicologia Hospitalar, pioneirismo e as pioneiras. In: Angerami-Camon, VA, organizador. 0 doente, a Psicologia e o hospital. 3a ed. São Paulo: Pioneira Thomson; 2001. p. 1-29.

3. Sebastiani RW. História e evolução da Psicologia da saúde numa perspectiva latino-americana. In: Angerami-Camon VA, organizador. Psicologia da saúde: um novo significado para a prática clínica. São PauIo: Pioneira Thomson Learning; 2002. p. 201-222.

4. Traverso-Yépez M. A interface Psicologia social e saúde: perspectivas e desafios. Psicol Estud. Maringá 2001; 6(2):49-56.

5. Pereira FM. A inserção do psicólogo no hospital ge ral: a construção de uma nova especialidade [dissertação]. Rio de Janeiro: Casa Oswaldo Cruz; 2003.

6. Sebastiani RW, Maia EM C. Contribuições da Psicologia da saúde-hospitalar na atenção ao paciente cirúrgico. Acta Cirur Bras 2005; 20(1):50-55.

7. Medeiros PF, Bernardes AG, Guareschi NMF. O conceito de saúde e suas implicações na prática psicológica. Psicologia: Teoria e pesquisa 2005; 21(3):263-269.

8. Romano BW. Entrevista: modelo para a prática da psicologia clínica em hospitais. Psi jornal de psicologia 1999 nov-dez [acessado 2012 dez 26]; 17(119) Disponível em: http://www.crpsp.org.br/portal/comunicacao/jornal_crp/119/frames/fr_entrevista. aspx

9. Chiattone HBC, Sebastiani RW. A ética em Psicologia Hospitalar. In: Angerami-Camon VA. A ética na saúde. São Paulo: Pioneira Thomson; 2002. p. 113139.

10. Kerbauy RR. Comportamento e saúde: doenças e desafios. Psicol USP 2002; 13(1):11-28.

11. Gorayeb R, Guerrelhas F. Sistematização da prática psicológica em ambientes médicos. Rev Bras ter comport cogn 2003; 5(1):11-19.

12. Romano BW. Equipe multiprofissional: é possível a inserção do psicólogo? In: Romano BW. Princípios para a prática da Psicologia clínica em hospitais. São Paulo: Casa do Psicólogo; 1999. p. 79-82.

13. Tonetto AM, Gomes WB. A prática do psicólogo hospitalar em equipe multidisciplinar. Estud Psicol (Campinas) 2007; 24(1):89-98.

14. M ota RA, M artins CGM , Véras RM. Papel dos profissionais de saúde na política de humanização hospitalar. Psicol Estud. 2006; 11(2):323-330.

15. Brasil. CNE/CES Resolução $n \circ 8$ de 7 de maio de 2004. Institui as Diretrizes Curriculares Nacionais para os cursos de graduação em Psicologia. Diário Oficial da União 2004; 18 maio.

16. Guaraná A. 0 dicionário biobibliográfico sergipano. Rio de Janeiro: Ed. Pongetti; 1925.

17. Vieira CMAM. A construção de um lugar para a Psicologia em hospitais de Sergipe [dissertação]. São Paulo: Pontifícia Universidade Católica de São PauIo; 2006.

18. Aguiar A, Medina E, Besnosik R, Vasconcelos S, Barreto T. A formação em Psicologia da saúde. CienteFico 2004; 1(4):1-14.
19. Bardin L. Análise de Conteúdo. Lisboa: Edições 70; 2004.

20. Minayo MCS. Fase de análise ou tratamento do material. In: Minayo MCS. 0 desafio do conhecimento. 3a ed. São Paulo, Rio de Janeiro: Hucitec, Abrasco; 1994. p. 197-247.

21. Vieira MJ. Suspensão de cirurgias: estudo exploratório em uma cidade do nordeste do país [dissertação]. Rio de Janeiro: Universidade Federal do Rio de Janeiro; 1982.

22. Straub RO. Introdução à Psicologia da saúde. In: Straub RO. Psicologia da Saúde. Porto Alegre: Artmed; 2005.

23. Bardagi MP, Bizarro L, Andrade AMJ, Audibert A, Lassance M CP. Avaliação da formação e trajetória profissional na perspectiva de egressos de um curso de Psicologia. Psicol Cienc Prof 2008; 28(2);304-315.

24. Campos GWS, Domitti AC. Apoio matricial e equipe de referência: uma metodologia para gestão do trabalho interdisciplinar em saúde. Cad Saude Publica 2007; 23(2);399-407.

25. Penna AG. Introdução à história da Psicologia contemporânea. 3 ed. Rio de Janeiro: Zahar; 1982.

26. Cecílio LCO. As necessidades de saúde como conceito estruturante na luta pela integralidade e equidade na atenção. Campinas: Departamento de M edicina Preventiva e Social da UNICAM P; 2001.

27. Cecílio LCO, Merhy EE. A integralidade do cuidado como eixo da gestão hospitalar. In: Pinheiro R, Mattos RA, organizadores. Construção da integralidade: cotidiano, saberes e práticas em saúde. 1 o ed. Rio de Janeiro: IMS, UERJ, ABRASC0; 2003. p. 197-210.

28. Cecílio LCO. O desafio de qualificar 0 atendimento prestado pelos hospitais públicos. In: M erhy $E E$, Onocko R, organizadores. Agir em saúde: um desafio para o público. 3o Ed. São Paulo: Hucitec; 2007. p. 293-319.

29. Brasil. Ministério da Saúde. Humaniza SU S. Brasília: M inistério da Saúde; 2009.

30. Barreto RA. Psicanálise e arte: o programa de humanização no hospital São Lucas em Sergipe. Estudos de psicanálise 2010; 33:137-146.

31. Fossi LB, Guareschi NMF. A Psicologia Hospitalar e as equipes multidisciplinares. Rev SBPH 2004; 7(1):29-43.

32. Motta $A B$, Enumo SRF. Brincar no hospital: estratégias de enfrentamento da hospitalização infantil. Psicol Estud. 2004; 9(1);19-28.

33. Moraes EO, Enumo SRF. Estratégias de enfrentamento da hospitalização em crianças avaliadas por instrumento informatizado. PsicoUSF 2008; 13(2): 221-231.

34. Portal da Psicologia OnLine (POL). Seminário de bate regulação e regulamentação das profissões de saúde no Brasil. 2010. [site na Internet]. [acessado 2012 fev 2]. Disponível em: http://www.pol.org.br/pol/ $\mathrm{cms} /$ pol/noticias/noticia_100818_001.html

35. Conselho Federal de Psicologia (CFP). I Fórum Nacional de Psicologia e Saúde Pública: contribuições técnicas e políticas para avançar o SUS. Brasília: Conselho Federal de Psicologia; 2006. 
36. Tonetto AM, Gomes WB. Competências e habilidades necessárias à prática psicológica hospitalar. Arq Bras Psicol 2007; 59(1):38-50.

37. Conselho Federal de Psicologia (CFP). Psicólogo brasileiro: práticas emergentes e desafios para a formação. Achcar R, coordenadora. São Paulo: Casa do psicólogo; 1994.

38. Kubo OM, Botomé SP. Formação e atuação do psicólogo para o tratamento em saúde e em organizações de atendimento à saúde. Interação Psicol. 2001; 5:93-122.

39. Abdalla IG, Batista SH, Batista NA. Desafios do ensino de Psicologia clínica em cursos de Psicologia. Psicol Cien Prof. 2008; 28(4):806-819.

40. Besteiro M M, Barreto MP. La formación de los profesionales de la salud: La contribuición del psicólogo hospitaleiro. In: Remor E, Arranz P, Ulla S, organizadores. El psicólogo em el âmbito hospitalario. Bilbao: Desclée de Brouwer Biblioteca de Psicologia; 2003.

Artigo apresentado em 17/03/2011

Aprovado em 11/04/2011

Versão final apresentada em 22/08/2011 